\title{
Diferencias entre el aprendizaje cooperativo y la asignación de tareas en la Escuela Rural. Comparación de dos estudios de caso en una unidad didáctica de acrosport en segundo ciclo de primaria \\ Differences between cooperative learning and practice style in Rural School. Comparison of two case studies in an acrosport didactic unit for the Second Cycle of Primary Education
}

\author{
José Juan Barba Martín \\ Universidad de Valladolid
}

\begin{abstract}
Resumen: En estas líneas se presenta una investigación cualitativa en una escuela de ámbito rural dentro de la asignatura de educación física. El objeto de esta investigación es comprobar si hay diferencias significativas en la enseñanza del acrosport en función de la metodología empleada: aprendizaje cooperativo y asignación de tareas. Para ello se han utilizado diferentes instrumentos, con el fin de triangular los datos. Estos han sido: ranking sociométrico, examen práctico, hoja de evaluación trimestral del alumnado, cuestionario motivacional, y cuaderno del profesor. Entre los resultados se podría observar una mejora en el ranking sociométrico, en el aprendizaje creativo y en la percepción de la participación en clase de los grupos de aprendizaje cooperativo. Habiendo casos individuales dentro de estos grupos en los que la aceptación por los compañeros y el sentimiento de aceptación por el aprendiz mejoran con el aprendizaje cooperativo. Estos resultados son similares al de otros estudios de una duración más amplia.
\end{abstract}

Palabra clave: Aprendizaje cooperativo, acrosport, socialización, motivación, estudio de caso.

Abstract: Through these lines it is presented a qualitative investigation in a rural school according to the Physical Education subject. The aim of this investigation is to check if there are any meaningful differences in the teaching of acrosport in accordance with the methodology used: cooperative learning and task assignment. To that effect, different instruments have been used in order to tally the information, which is: a ranking to see the social relationships among students, a practical exam, a pupil's term evaluation sheet, a motivational question paper and a teacher's notebook. Among the results, it could be observed an improvement in the social relationships ranking, in the creative learning and in the perception of the participation of cooperative learning groups. There are some individual facts in these groups where the acceptance from partners and the sense of acceptance from the learner improve with the cooperative learning. These results are similar to other studies which are longer.

Key words: Cooperative learning, acrosport, socialization, motivation, case study.

\section{Introducción}

Los nuevos tiempos están transformando la realidad educativa hasta el punto de que la memoria, lo más valorado en la escuela, apenas tiene sentido para la vida actual (Bauman, 2005). Esto es debido a que nos encontramos en un mundo en continuo cambio, ya nada es estático, ya no sirve acumular conocimientos que algún día podrán resultar válidos. En la actualidad todo tiene fecha de caducidad. Diferentes autores (Bauman, 2000; Ovejero Bernal, 2004; Muniesa, 2008;...), reconocen que estos cambios están favoreciendo un individualismo feroz, que está desmontando la sociedad del bienestar.

Desde el mundo de la educación no sólo debemos educar para éstos, sino que hemos de presentar una postura crítica ante ellos. No podemos considerar al estudiante como un consumidor en potencia, sino que ha de constituirse como una persona crítica, integrada dentro de una red de relaciones sociales que se desarrolla en la medida en que se relaciona con los demás (García Carrasco, 2007).

El planteamiento de este estudio parte de la idea de Giroux (2008) de no considerar la educación como neutral, ya que en caso de hacerlo así lo que se hace es adoctrinar. Desde esta perspectiva crítica de la educación, Ovejero Bernal (2004) plantea tres cambios que se deben introducir en la escuela como una forma de mejorar la sociedad ante los nuevos tiempos, siendo estos: Aumentar la capacidad de autonomía y el pensamiento crítico de los ciudadanos; Incrementar el grado de apoyo social y mejorar la integración en redes sociales; Mejorar nuestras habilidades de cooperación desarrollando nuestros sentimientos y conductas de solidaridad.

Diversos autores (Ovejero Bernal, 1990, 2004; Velázquez Callado, 2004; Fernández-Río, 2003. ..) consideran que el aprendizaje cooperativo

Fecha recepción: 19-10-09 - Fecha envío revisores: 19-10-09 - Fecha de aceptación: 24-03-10 Correspondencia: José Juan Barba Martín

Escuela de Magisterio de Segovia.

Plaza de Colmenares, 1.40001 Segovia

E-mail: jjbarba@gmail.com
(AC) proporciona respuestas en la línea de mejorar la autonomía, mejorar la socialización y la solidaridad. Ovejero Bernal (1993), considera que las técnicas de AC, se basan en los principios interaccionales, favoreciendo la construcción social de la inteligencia, la motivación intrínseca, el nivel de aspiración o el autoconcepto. Pero el AC no es un método que sólo se basa en el aprendizaje de aspectos sociales, sino que asegura que ya está legitimado al ser eficaz a la hora de desarrollar también los aprendizajes tradicionales (Cohén, 1994;Barret, 2005; Dyson, 2001, 2002; Johnson y Johnson, 1994) y en una fuerte conexión entre los aprendizajes teóricos y prácticos (Casey, Dyson, y Campbell, 2009). Dentro de esta misma línea, Velázquez Callado (2004) considera que los docentes que optan por el trabajo cooperativo pretenden que, además de que sus estudiantes logren los objetivos de aprendizaje propios del área, aprendan a ayudarse mutuamente, sean capaces de trabajar en grupo autónomamente, desarrollen habilidades sociales, se autorregulen en los conflictos y desarrollen aspectos afectivos hacia sus compañeros.

En el área de educación física (EF) nos encontramos con diversos estudios relacionados con el AC. Entre estos destacan: Dyson (2001), que después de dos programas de AC de ocho sesiones cada uno, basados en habilidades y destrezas básicas, observó que la mejora en el rendimiento de las habilidades motrices había sido similar al de cursos anteriores con una metodología más directiva. Sin embargo, se muestra una mejora en las habilidades sociales de los estudiantes, como son: la confianza, la capacidad de animar, la amistad... En otro trabajo Fernández-Río (2002) después de un programa anual basado en metodología cooperativa, concluye que: mejora la autoestima, el autoconcepto, la honestidad, la responsabilidad; favorece la interacción entre los compañeros; y responsabiliza más a los estudiantes. Además, dentro del área de EF: aumenta la valoración de la habilidad, de la apariencia, de la destreza en los deportes; favorece la motivación hacia la clase; y aumenta el gusto de los alumnos por la actividad física. Por último, Velázquez Callado (2006) recoge un estudio de 15 sesiones referentes al AC del salto de comba. En él, se hace referencia a que los estudiantes alcanzaron las competencias motrices previstas en un ambiente en el que apenas hubo conflictos; $y$ se favorecieron situaciones 
de ayuda, de ánimo y de aceptación por el grupo de compañeros. Esto se completó con una gran motivación hacia la actividad que llevó a su práctica fuera de la escuela.

Como conclusión a estos estudios en EF, se podría afirmar que, el AC permite un nivel de adquisición de contenidos similar a los métodos de aprendizajes tradicionales, pero con una mejora en el desarrollo de habilidades sociales. Si bien, no existen referencias sobre que sucede cuando nos enfrentamos al tratamiento del AC en una duración inferior a 15 sesiones.

La preocupación de esta investigación es quésucede cuando solose trabaja de forma cooperativa en una unidad didáctica de un mes de duración, si los resultados propios del AC se comienzan a observar, o si por el contrario es necesario más tiempo para observar cambios. Concretando más, este trabajo consiste en comprobar si durante un mes, con dos sesiones a la semana se observa alguna diferencia significativa entre los aprendizajes en un mismo contenido en un grupo con enseñanza tradicional, más concretamente con asignación de tareas (AT) y otro con AC.

\section{Material y método}

\subsection{Contextualización y selección de los grupos}

Este estudio se ha llevado a cabo en un Centro Rural Agrupado (CRA) durante ocho sesiones en una unidad didáctica de acrosport, con estudiantes de segundo ciclo de primaria sin experiencia previa en este contenido, pese a que según recogen Vernetta Santana, et Al. (2008) son numeroso autores los que lo consideran un contenido importante en educación física por sus aspectos cooperativos, estéticos y creativos.

Debido a la baja matriculación, estos estudiantes se encuentran en un mismo grupo clase la mayoría del tiempo, si bien en determinadas áreas la clase se desdobla por cursos. Este es el caso del área de EF, que se imparte de forma separada. El primer grupo, es del curso de $3^{\circ} \mathrm{de}$ primaria con 9 estudiantes, siendo 3 niñas y 6 niños. El segundo grupo, tiene 7 estudiantes de $4^{\circ}$ de primaria, dividiéndose en 2 niñas y 5 niños. En ambos cursos, la media de la competencia motriz de los estudiantes es muy buena, definida por el maestro de educación física y corroborada por las calificaciones trimestrales y las anotaciones en los expedientes del alumnado. Si bien, en cada curso se encontraba una niña con muchas dificultades en las prácticas del área. Respecto al comportamiento, hay que tener en cuenta la opinión de los maestros que consideran a ambos grupos como disruptivos y con facilidad para que surjan conflictos entre los estudiantes.

Una vez definidos los grupos, el siguiente paso era saber a qué grupo le iba a corresponder el AC y a cual el estilo de AT; y cuáles iban a ser los subgrupos de trabajo. La asignación de cada tipo de aprendizajes se realizó mediante un sorteo, dando como resultado que el grupo de tercer curso trabajaría mediante AC y el grupo de cuarto mediante AT. Posteriormente había que decidir los subgrupos de trabajo, teniendo en cuenta que los grupos de AC son útiles en la medida en que todos los participantes se encuentran implicados y con una tarea definida, siendo un valor positivo la heterogeneidad en los grupos (Ovejero Bernal, 1990). A esto hay que añadir que el acrosport en estas edades se ha de plantear desde figuras sencillas. Ante estas razones se optó porque los grupos fueran pequeños, de tres estudiantes menos uno de cuatro en el grupo de AT, manteniéndose estables durante toda la unidad didáctica.

\subsection{Metodología del trabajo en el aula}

Una de las decisiones importantes era diferenciar en qué consistía el AC, y en qué los métodos tradicionales, con el fin de tener muy clara la actuación en el aula. Esto se concretó en las siguientes pautas de actuación.

El grupo que aprendió acrosport mediante métodos tradicionales se basó en la enseñanza por asignación de tareas, denominada en este texto AT (Mosston y Asworth, 2001). Sus principales características son: que la tarea es fija y debe ejecutarse según un modelo específico, y puede evaluarse en correcto o incorrecto. Esta técnica se aplicó de la siguiente forma:
-Propuesta de aprendizaje. Se entregaba una ficha con una figura de acrosport con la técnica que tenían que aprender. Reunidos todos en corro, el maestro explicaba la tarea y se hacía una demostración. Antes de comenzar a trabajar, el maestro asignaba los diferentes roles a cada miembro del grupo.

-Fase de trabajo. Durante 10 minutos los estudiantes repetían incesantemente la figura, con el fin de conseguir una ejecución perfecta. El rol del profesor consistió en ir por los grupos haciendo correcciones técnicas del trabajo que se estaba desarrollando.

·Exhibición. Pasado el tiempo de trabajo, cada grupo presenta a los compañeros lo realizado. Esto sirve por un lado para poner fin a la actividad, y por otro como forma de evaluar los aprendizajes de cada grupo.

El grupo que desarrolló el aprendizaje del acrosport a través de estrategias cooperativas, se basó en la técnica learning together (Johnson y Johnson, 1994). Ésta consiste en que los estudiantes dominen los contenidos y que a la vez ayuden a sus compañeros en el aprendizaje, trabajando todos los miembros del grupo en una tarea única. Para Goicoetxea Iraola y Pascual Hoyelos (2002) es el método más cercano al aprendizaje puramente cooperativo, pero a la vez resulta difícil valorar la contribución de cada participante. Si bien, en este caso no es un problema, ya que si las contribuciones de todos no son positivas, no se pueden construir las figuras:

La puesta en práctica de esta estrategia se concretó en los siguientes pasos:

Propuesta de aprendizaje. A los estudiantes se les enseñaba una figura de acrosport, con una técnica que debían aprender. La aplicación de la técnica era obligatoria, si bien tenían que inventar el resto de la figura. Esto pretendía fomentar la solución de problemas y el aprendizaje divergente, principales características de esta técnica (Ovejero Bernal, 1990).

-Trabajo grupal. Cada grupo tenía 10 minutos para ensayar y preparar su figura. Durante este tiempo dialogaban y tomaban sus propias decisiones, siendo necesario que una vez que se pusieran de acuerdo, presentaran el plan de trabajo de forma oral al maestro, en él tenían que incluir qué iban a hacer y cuál era el rol de cada estudiante.

- Con el fin de que los estudiantes pudieran autoevaluarse y mejorar su percepción del trabajo tenían la posibilidad de pedir al maestro que les hiciera una foto digital. Esto permitía tener una prueba visual de lo que habían estado haciendo y debatir sobre errores o nuevas posibilidades de trabajo.

- El rol del profesor quedaba limitado a asesorar a través de preguntas muy generales que no llevaran implícita una respuesta. Ya que en el AC los profesores no deben intervenir más allá de lo estrictamente necesario (Ovejero Bernal, 1990).

-Exhibición. Pasado el tiempo de trabajo, cada grupo presenta a los compañeros el trabajo realizado. Esto servía como forma de enseñar a los compañeros el trabajo realizado.

Este planteamiento reúne los principios necesarios para poder considerar el aprendizaje como cooperativo (Ovejero Bernal, 1990; 2003), siendo estos: Interdependencia positiva, interacción cara a cara entre los estudiantes, responsabilidad individual, y utilización por parte de los miembros del grupo de habilidades interpersonales y grupales.

\subsection{Metodología e Instrumentos de medición}

En esta investigación se ha optado por una metodología cualitativa, que permita conocer en profundidad la realidad educativa en la que se realizó la investigación, y los efectos que se presentaron. A la par que buscar la transferencia en otras situaciones similares.

La investigación cualitativa es una actividad sistemática orientada a la comprensión en profundidad de los fenómenos educativos y sociales, a la transformación de prácticas y escenarios socioeducativos, a la toma de decisiones y también al descubrimiento de un cuerpo organizado de conocimientos (Sandín, 2003: 123).

Para ello se ha optado como método de investigación por el estudio de caso. Para Zabalza Beraza (2002) este método, intenta comprender: 
al individuo o grupo, su situación y comportamiento en la configuración total de factores que le afectan a través del tiempo. También se caracteriza porque no parte de una hipótesis, sino que se indaga en la realidad (Sánchez Arroyo et Al., 2008). De este modo, para Stake (1999) la investigación cualitativa recopila información queva a teneruna aplicación directa para el caso estudiado, del mismo modo que también tiene elementos en común y transferencia con otros casos similares. (Stake, 1999).

Para la selección de los instrumentos ha sido muy importante recoger información desde múltiples puntos de vista, con el fin de triangularlas. Para ello se han utilizado los siguientes instrumentos:

-Ranking sociométrico: Los estudiantes tenían que valorar la relación con todos sus compañeros de curso respecto a los siguientes ítems: muy mal, mal, regular, bien, muy bien. Estas valoraciones se tradujeron auna escalanumérica graduada (muy mal=1; muy bien=5) loquepermitió calcularuna puntuación individual por cada estudiante y una general para cada grupo-clase. Esta prueba se realizó de forma previa y posterior al trabajo, con el fin de poder valorar los cambios producidos.

-Examen práctico. Después de terminar las ocho sesiones de aprendizaje, se realizó un examen, consistente en la ejecución de cuatro figuras. Para ello, se les entregó una hoja con dos figuras a imitar (trabajo realizado por el grupo de AT) y otras dos técnicas para ejecutar, teniendo que completarlo desde su concepción creativa (trabajo realizado por el grupo de AC). Una vez entregada la documentación, tuvieron 15 minutos para prepararlas, pasado este tiempo realizaron las 4 figuras delante de un maestro ajeno a la investigación, pero con conocimientos en acrosport. Éste valoró las diferentes figuras de acuerdo a unos ítems cerrados (calidad, aplicación de la técnica, velocidad de ejecución, coordinación y creatividad) Valorándolos de 1 (muy mal) a 5 (muy bien).

-Hoja de evaluación trimestral. Al finalizar el trimestre cada estudiante realiza una ficha en la que tiene que expresarse sobre diferentes cuestiones abiertas. Estas son: quéhan aprendido, lo qué más les ha gustado, lo qué menos, cuál ha sido la relación con sus compañeros, cómo se han sentido, y cuál ha sido su relación con el maestro. Este cuestionario era el mismo que se pasa todos los trimestres, sin preguntas específicas sobre el trabajo de acrosport. -Cuestionario sobre motivación. Fue aportado por el orientador del centro, siendo la prueba con la que mide la motivación en las diferentes áreas. Si bien, se matizó la redacción sustituyendo el término «clases», por «clases de acrosport» con el fin de evitar malas interpretaciones por los estudiantes. En él había diferentes preguntas que hacían hincapié en tres bloques diferentes: la atención, la distracción, y la participación activa e iniciativa en la clase.

-Cuaderno del profesor. Lo llevaba el maestro realizando un registro de las tareas llevadas a cabo en clase, de los diferentes conflictos surgidos y de las dificultades encontradas. Además, servía como instrumento de recogida de datos de las opiniones de los estudiantes en los diferentes momentos de diálogo.

\section{Análisis de datos}

-Ranking sociométrico (tabla 1): Los resultados en este apartado fueron los siguientes:

Inicialmente el grupo de AC obtuvo una media grupal de 3'42. En cuanto a los estudiantes nos encontramos con un estudiante por debajo de la puntuación mal (2), además de otros tres por debajo de regular(3). También aparecen cuatro estudiantes con la valoración bien(4) o superior.

Este grupo al terminar el trabajo consiguió una puntuación media en el ranking sociométrico de 3’93, incrementándose la puntuación inicial en 51 centésimas. En cuanto a las valoraciones individuales al acabar el trabajo no hubo ningún estudiante por debajo de la valoración mal, 2 por debajo de la valoración regular, y 4 estudiantes por encima dela valoración bien.

Entre los estudiantes que mayor cambio produjeron en su valoración se encuentran una estudiante (Marta) que pasó de la valoración 1’71 a
3, y otra (Carmen) que pasó de 2’42 a 3. También son destacables un estudiante que pasó de 4'42 a 4'87 y otro que pasó de 4'28 a 4'85. El resto, o se mantuvieron las puntuaciones, o no se modificaron positivamente más de 15 centésimas, pero en ningún caso descendieron.

Al inicio del proceso el grupo de AT consiguió una media de la clase de 3’90. Ningún estudiante se encontraba por debajo de regular (3) y dos estudiantes se encontraban por encima de bien (4).

Respecto a los estudiantes de forma individual, nos encontramos a dos por debajo de regular (2'67 y 2'83), y en el lado positivo hay dos estudiantes con una valoración superior a bien (4'17 y 4’5).

Al terminar el trabajo el grupo consiguió una puntuación media de 3’57, empeorando en 33 centésimas la primera valoración.

En este grupo no hay ningún estudiante que haya mejorado su valoración, si bien hay dos que la han mantenido (4’5 y 3’83). Entre los estudiantes que mayor valoración perdieron, más de medio punto, se encuentran los siguientes casos: de 3'33 a 2'67, de 3'83 a 3'33, y de 3'67 a 2'83,. Los otros dos estudiantes del grupo registraron una pérdida de menos de 20 centésimas.

-Examen práctico (tabla 2). Los datos del examen han sido procesados de forma global en función del tipo de aprendizaje de cada grupo, haciendo la media de todos los subgrupos que le forman. El curso que utilizó el AC ha obtenido una puntuación media de 4'38; mientras que el que se basó en AT ha obtenido una puntuación media de 3'62.

Respecto a la valoración por los bloques del examen, en las dos primeras preguntas, que correspondían a la forma de trabajo del grupo deAT, el grupo de AC consiguió 4'37, mientras el grupo deAT 4'25. En la segunda parte, con las dos actividades de la forma de trabajo del grupo de AC, el grupo que utilizó este aprendizaje consiguió una puntuación media de 4’4, mientras que el grupo de AT consiguió 3.

·Hoja de evaluación trimestral. Sibien no hapresentado preguntas referentes a acrosport, los estudiantes sí que ofrecieron respuestas significativas en sus contestaciones. Entre ellas nos encontramos:

Dentro del grupo de AC, siete de nueve estudiantes consideran que el acrosport ha sido el contenido que más le ha gustado durante el trimestre. Estos estudiantes también consideran que uno de los aprendizajes del trimestre es que se llevan mejor con sus compañeros. Por ejemplo la siguiente cita de Jaime «Tengo más amigos por hacer acrosport en EF». Cinco de estos estudiantes hacen referencia a que se han sentido muy a gusto en clase, un ejemplo es la cita de Santiago «Me he sentido muy bien con mis compañeros, con Jaime mejor que nunca» Si bien ha habido un estudiante (Pedro) que expone que no se ha sentido bien en clase, ya que lo peor del trimestre ha sido oír protestar a Manuel durante las sesiones de acrosport.

Dentro de este grupo cabe destacar las aportaciones de dos estudiantes. Carmen considera que «sólo me he sentido bien con mis compañeros del grupo de acrosport»; y Marta. Considera que en las sesiones lo ha pasado «bien porque cada vez me voy haciendo más amiga de todos», siendo lo que más le ha gustado «que ya no me insultan».

\begin{tabular}{|c|c|c|c|}
\hline \multicolumn{4}{|c|}{ Tabla 1. Ranking sociométrico grupal (valorado entre 1 y 5) } \\
\hline & Previo a la UD & $\begin{array}{c}\text { Posterior a la } \\
\text { UD }\end{array}$ & Variación \\
\hline Grupo AC & $3 ’ 42$ & 3’93 & $+0 ’ 51$ \\
\hline Grupo AT & 3990 & $3 \prime 57$ & $-0,33$ \\
\hline \multicolumn{4}{|c|}{ Tabla 2. Resultados del examen (valorado entre 1 y 5) } \\
\hline & $\begin{array}{l}\text { Pruebas de } \\
\text { imitación }\end{array}$ & $\begin{array}{l}\text { Pruebas de } \\
\text { creación }\end{array}$ & Media \\
\hline Grupo AC & 4,37 & 4,40 & 4,38 \\
\hline Grupo AT & 4,25 & 3.00 & 3,62 \\
\hline
\end{tabular}

\begin{tabular}{|l|c|c|c|}
\hline \multicolumn{4}{|c|}{ Tabla 3. Resultados del cuestionario sobre la motivación (valorado entre 1 y 5) } \\
\hline & $\begin{array}{c}\text { Preguntas de } \\
\text { atención }\end{array}$ & $\begin{array}{c}\text { Preguntas sobre } \\
\text { distracción }\end{array}$ & $\begin{array}{c}\text { Preguntas sobre } \\
\text { participación en clase }\end{array}$ \\
\hline Grupo AC & 4,38 & 1,88 & 3'92 \\
\hline Grupo AT & 4,15 & 1,44 & 2 '00 \\
\hline
\end{tabular}


En el grupo del AT hay tres estudiantes de los siete que consideran el acrosport como su contenido favorito del trimestre. Ningún estudiante ha hecho referencia a que el acrosport le haya proporcionado una mejora en la amistad o en el aspecto emocional. Si bien, si que hay dos (Orlando y Cayetano) que consideran que la relación con sus compañeros no ha sido buena porque se insultan.

Cuestionario sobre la motivación en el trabajo con acrosport (tabla 3). En las preguntas referidas a la atención y a la distracción, apenas se muestran diferencias significativas entre ambos grupos. Las referidas a la atención se aproximan a 4'5 y las referidas a la distracción también muestran una media de 1'5. Con lo que todos los estudiantes valoran su atención entre buena y muy buena, y su distracción entre baja y muy baja.

Las diferencias entre los grupos se encuentran en las preguntas que hacen referencia a la participación activa en clase. Mientras que el grupo de AC se valora en 3'92 sobre 5, el deAT lo hace en 2. Así en la pregunta «He tomado parte en las discusiones o actividades que se realizan en clase de acrosport» en el grupo de AC se valora con 4'22; mientras que en el de AT se valora como 2’57. En la pregunta «En ocasiones soy yo el que he expuesto a mis compañeros el trabajo a realizar en clase de acrosport» los estudiantes del grupo de AC se valoran con 3'78, mientras que los de AT se valoran en 2'28. Por último, a la cuestión «El grupo ha realizado trabajos extra por mi propia iniciativa», el grupo de AC se valora en 3'78, mientras que el de AT lo hace en 1'14, siendo 1 la puntuación mínima y 5 la máxima.

-Cuaderno del profesor. En él se registraron principalmente los conflictos surgidos y las dificultades encontradas.

Dentro del grupo de $A C$, nos encontramos con que en la segunda sesión el subgrupo G1AC, no quiso trabajar porque había problemas personales entre los participantes que les hacían no querer interaccionar juntos. Se optó por la solución autónoma del conflicto, y como esta no llegó se habló entre toda la clase para convencerles de que aunque tuvieran problemas personales debían trabajar en grupo, solucionándolo de esta forma. Sobre este grupo no hay más registros sobre conflictos. Si bien, sí que hay recogido un comentario de Marta en la sesión 7 en la que dice que «ya no se enfadan tanto porque hay que trabajar juntos después».

En el subgrupo G2AC no hubo ningún conflicto. No obstante, en la tercera sesión Santiago dice que está contento de trabajar con Jaime, que ahora se lleva mejor con él. A partir de este momento repite este comentario en numerosas ocasiones. Estos comentarios se ven reflejados en el ranking sociométrico y en la hoja de evaluación trimestral.

El subgrupo G3AC tiene registrados cinco conflictos, en las sesiones 2, 3, 4, 5 y 6. Los problemas siempre estaban derivados de ver que persona ocupa cada rol. Mientras que los demás grupos siguen un criterio de peso y fuerza para elegir las posiciones de cada miembro, en este grupo el criterio de elección estaba basado en que posición les gusta más. El maestro les sugiere que todos prueben todas las posibilidades y busquen como lo hacen mejor, de este modo resuelven el conflicto, además de servirles como una guía de actuación en el resto de sesión, si bien en la sesión siguiente vuelven a discutir por lo mismo. A partir de la sesión 6 no se vuelve a repetir este conflicto.

A nivel general de todos los grupos hay que destacar que al acabar las dos últimas sesiones los estudiantes protestan porque «se les ha hecho muy corta la hora», proponiendo: «podríamos seguir un poco más».

En el grupo de aprendizaje basado en el AT, el G1AT comienza a trabajar de manera muy eficaz y a partir de la cuarta sesión comienzan a tardar muy poco tiempo en hacer las figuras con bastante corrección. El buscar matices a la presentación es una tarea que les aburre, quedando este comentario recogido en todas las tareas a partir de la cuarta sesión.

El G2AT registra numerosos conflictos en todos los ejercicios. El que se da en todas las prácticas es que Orlando y Cayetano, no trabajan con cuidado lo que provoca pequeños accidentes o dificultan trabajar a sus compañeros. Estos protestan llegando un par de ocasiones a pedir al maestro el cambio de grupo. Los conflictos no se resuelven nunca, independientemente de regañinas o de la separación temporal de la actividad, antes o después se continúa repitiendo el comportamiento. En este subgrupo surge otro conflicto, ya que Cristina no es capaz de realizar sola determinadas posturas. Sus compañeros en lugar de ayudarla, se quejan al maestro de que no puede hacerlo. Esto no se soluciona y se sigue produciendo desde la sesión tercera hasta el final.

También queda registrado que durante el tiempo de preparación de los ejercicios el día del examen los grupos de AT se encuentran inseguros ante la preparación de las pruebas, pidiendo aprobación al maestro; si bien este no puede dársela debido a las características de la prueba.

\section{Discusión.}

\section{Socialización}

Dentro de los dos grupos, se puede observar una modificación considerable en el ranking sociométrico del grupo que trabajó mediante el AC, incrementándose la valoración media en más de medio punto. El caso contrario se produjo en el grupo de AT que disminuyó su valoración en 33 centésimas. Este cambio se ve ratificado por los datos que aportan en las hojas de evaluación trimestral, en las que hay estudiantes de AC que se sienten más valorados y ven como mejoran las relaciones con sus compañeros. Es importante la doble dirección de estos cambios. Por un lado, la perspectiva de los compañeros respecto al estudiante con poca valoración vista en el ranking sociométrico, y por otro, la de los propios interesados que lo valoran en la hoja de evaluación trimestral. Esto supone que el cambio es evidente, en la línea de otros trabajos (Grineski, 1993; Dyson, 2001, 2002; Fernández-Río, 2003; Velázquez Callado, 2004; Casey, Dyson y Campbell, 2009; Metzeler, 2005...).

Hay que tener en cuenta que las mejoras significativas en la valoración de los compañeros, sólo se ha producido en cuatro casos, provocando un incremento notable en la valoración media del grupo, como queda reflejado en el ranking sociométrico. Esto podría estar condicionado por la duración dela experiencia, ya que en las tres relatadas en la introducción, el período de puesta en práctica es de al menos 15 sesiones, el doble de tiempo que el dedicado a este trabajo. También hay que tener en cuenta que para que se observen cambios significativos dentro de los grupos, Ovejero Bernal (1990), hacereferencia a que los grupos deben permanecer trabajando juntos al menos un trimestre y, si es posible, todo el curso.

\section{Calidad del trabajo}

Respecto a la calidad del trabajo, medida por un examen, llama la atenciónque la diferencia cuando es una postura aimitarno es significativa, estando los dos grupos al mismo nivel. Sin embargo, si además de realizar eficazmente la tarea medimos el grado de creatividad, nos encontramos con que el grupo de AC consigue un mejor resultado, de 1'4 puntos. Se podría considerar que este cambio, es debido al tipo de trabajo realizado por los diferentes grupos. Cuando el examen solo se centra en ejecutar, ambos grupos se muestran eficaces; sin embargo, sí hay que crear sobre una técnica, el grupo de AC se muestra más capacitado. Esto se ve respaldado por los datos del cuestionario sobre motivación, en el que los estudiantes del grupo de AC, valoran su participación en las clases como más activa y consciente, y por la anotación del cuaderno del maestro en la que observa el día del examen que el grupo de AT se muestra inseguro en la realización de la parte creativa. En este sentido hay diferentes estudios en el campo del aprendizaje en EF, que consideran que la calidad del trabajo es igual o superior que con otros métodos (Casey, Dyson, y Campbell, 2009; Barret 2005, Dyson 2001, 2002; Fernandez-Rio, 2003,...).

Estos datos corroboran las pautas indicadas por Vernetta el Al. (2008) para el desarrollo de una buena composición desde el punto de vista de la creatividad en acrosport. Así, en el grupo de AC se ha tenido en cuenta los criterios técnicos imprescindibles para la realización de este contenido y se ha dejado abierto a los estudiantes la intuición motora cooperativa-interpretativa, la inspiración y la sensibilidad artística por parte de los estudiantes para su composición. Mostrando esta metodología unos resultado positivos.

\section{Motivación}

Respecto a la motivacióny a la distracciónno semuestran diferencias entre los dos grupos. Ambos grupos definen su nivel de motivación 
como muy alto, y la distracción como muy baja, siendo ésta una de las principales características del AC (Ovejero Bernal, 1990; Velázquez Callado, 2004; Fernández-Río; 2003; Prieto Navarro, 2007), no obstante en el cuaderno del profesor se registra que uno de los grupos de AT, enseguida acaba las tareas y pierde el tiempo. Pero no podemos entrar a valorar la percepción personal de los estudiantes, y si consideran motivante hacer pronto y bien las tareas, aunque les sobre tiempo.

Casos individuales

Uno de los aspectos más interesantes de este estudio, puede considerarse el análisis de dos casos particulares. El primero es el de la estudiante de AC Marta, que con una valoración inicial en el ranking sociométrico de 1’71, pasó a una valoración de 3, lo que supone un incremento muy significativo en la valoración por sus compañeros. Marta contestó en el cuestionario de evaluación que «se siente más amiga de todos y que ya no la insultan». Además, en el cuaderno del profesor no se recogen ni conflictos ni reproches en su grupo. Pese a su clara torpeza motriz, ha conseguido participar de todas las tareas y mejorar su rendimiento, consiguiendo su grupo una puntuación similar a la de los de AC en el examen.

El otro caso a tener en cuenta es el de Carmen, que pasó en el ranking sociométrico de 2’42 a 3, siendo aceptada en su grupo, y considerando en el cuestionario de evaluación trimestral que el acrosport ha sido su contenido favorito. Esto se ve reflejado en el cuaderno del profesor, recogiendo que participó todo el tiempo de las sesiones, siendo esto especialmente relevante ya que es una estudiante que tiene mucha reticencia a participar en actividades grupales.

Podría considerarse que en ocho sesiones se comienzan a observar los cambios que se definen en la literatura: mejora la percepción social del grupo de compañeros, mejora la participación en clase, alta motivación, mayor creatividad y similar nivel de aprendizajes; dándose todos estos cambios a favor del grupo de AC. Si bien, puede considerarse que los cambios principales son los que afectan a nivel individual. En el caso de Marta y Carmen ha servido para que dos estudiantes, poco valoradas socialmente, se hayan sentido integradas y valoradas por su grupo, a la par que su grupo les ha reconocido como tales. Es entonces cuando esta experiencia sobre AC cobra sentido, cuando aporta dignidad al ser humano.

Los niños/as de nuestras clases pueden pasar perfectamente sin muchos de los contenidos, actividades y pruebas del actual currículum de EF. Sin embargo, el reconocimiento de su Dignidad lo necesitan como el aire que respiran. No se trata, pues, de que simplemente aprendan una determinada destreza o habilidad, sino de que se sientan dignos a través de ella (no indignos por no tenerla) (Fernández-Balboa, 2004: 224).

$\underline{\text { Notas. }}$

Los estudiantes se nombran siempre con el mismo seudónimo, siendo interesante para comprobar como se perciben los cambios en los diferentes instrumentos de investigación.

\section{Bibliografía.}

Barret, T. (2005). Effects of cooperative learning on the performance of sixth-grade physical education pupils. Journal of Teaching in Physical Education. 24, 88-102.

Bauman, Z. (2005). Los retos de la educación en la modernidad líquida. Barcelona: Gedisa.

Bauman, Z. (2000). Trabajo, consumismo y nuevos pobres. Barcelona: Gedisa.

Casey,A.; Dyson, B.; y Campbell,A. (2009). Action research in physical education: focusing beyond myself through cooperative learning. Educational Action Research. 17, (3), 407-423.

Cohén, E. (1994). Restructuring the classroom: Conditions for productive small groups. Review of Educational Research, 64, 1-35.

Dyson, B. (2002). The implementation of cooperative learning in an elemntary physical education program. Journal of Teaching in Physical Education. 22, 69-85.
Dyson, B. (2001). Cooperative learning in an elementary Physical Education program. Journal of Teaching in Physical Education. 20, 264-281.

Fernández-Balboa, J. M. (2004). La Educación Física desde una perspectiva crítica: de la pedagogía venenosa y el currículo oculto hacia la dignidad. En: VM. López Pastor; R. Monjas Aguado; y A. Fraile Aranda, (Coords.). Los últimos diez años de Educación Física Escolar (215-225). Valladolid: Universidad de Valladolid.

Fernández-Río, J. (2002). La autoestima, la educación física actual y el aprendizaje cooperativo. En: Libro de actas del VI congreso Estatal de Actividades Físicas cooperativas. Valladolid: La Peonza.

Fernández-Río, J. (2002). La autoestima, la educación física actual y el aprendizaje cooperativo. Libro de actas del VI congreso Estatal de Actividades Físicas cooperativas. Valladolid: La Peonza.

García Carrasco, J. (2007). Leer en la cara y en el mundo. Barcelona: Herder.

Giroux, H.A. (2008). Democracia, educación y política en la pedagogía crítica. En: P. McLaren; y JL. Kincheloe(Eds.). Pedagogía Crítica. (17-22). Barcelona: Grao.

Goicoetxea Iraola, E.; Pascual Hoyelos, G. (2002). Aprendizaje cooperativo: bases teóricas y hallazgos empíricos que explican su eficacia.Educación XXI, 5, 199-226.

Grineski, S. (1993): Achieving educational goals in physical education. A missing ingredient. Journal of Physical Education, Recreation and Dance, 64 (5), 32-34.

Johnson, D.W. y Johnson, R.T, (1994). Learning together and alone: Cooperative, competitive, and individualistic learing (4. ed.). Needham Heights, MA: Allyn \& Bacon.

Mosston, M.y Ashworth, S. (2001). La enseñanza de la educación física. Barcelona: Hispano Europea.

Muniesa, B. (2008). Libertad, Liberalismoy democracia. Barcelona: El viejo topo.

Ovejero Bernal,A. (2004). Globalización, sociedad y escuela. Valladolid: Universidad de Valladolid.

Ovejero Bernal, A. (2003). Aprendizaje Cooperativo: Una escuela diferente para una sociedad diferente. En:A. Guil (Coord.). Psicología social del sistema educativo: Líneas de trabajo e investigación, (71-90). Sevilla: Kronos.

Ovejero Bernal, A. (1993). Aprendizaje cooperativo: Una eficaz aportación de la psicología social a la escuela del siglo XXI. Psicothema. 5(Extra 1)373-391.

Ovejero Bernal,A. (1990). El aprendizaje cooperativo. Una alternativa eficaz a la enseñanza tradicional. Barcelona: PPU.

Prieto Navarro, L. (2007). El aprendizaje cooperativo. Madrid: PPC.

Sánchez Arroyo, J.F.; Chinchilla Minguet, J.L.; de Burgos Carmona, M.; y Romero Ramos, O. (2008). Las relaciones sociales y educativas existentes entre los elementos personales del proceso educativo durante una sesión de Educación Física. Un estudio de casos. Retos. 14, 66-69.

Sandín, M.P. (2003). Investigación cualitativa en educación. Madrid: McGraw-Hill.

Stake, R.E. (1999). Investigación con estudio de casos. Madrid: Morata.

Velázquez Callado, C. (2006). Aprendemos juntos a saltar a la comba. Una experiencia de aprendizaje cooperativo en educación física. En: Actas del V Congreso Internacional de Actividades Físicas Cooperativas. Valladolid: La peonza.

Velázquez Callado, C. (2004). Las actividades físicas cooperativas. Una propuesta para la formación en valores a través de la educación física en las escuelas de educación básica. México: Subsecretaria de Educación Básica y Normal.

Vernetta Santana, M.; López Bedoya, J.; y Gutiérrez Sánchez, A. (2008). La creatividad en gimnasia acrobática. En: A. Martínez Vidal; y P. Díaz Pereira (Coord.). Creatividad y deporte: Consideraciones teóricas e investigaciones breves. (133-154). Sevilla: Wanceulen.

Zabalza Beraza, M.A. (2002). Diarios de clase. Madrid: Narcea. 